\title{
Heterodyne polarimetry technology for inspection of critical dimensions
}

\author{
V. Protopopov
}

Samsung, APT4, 405-1002, Suwon City, South Korea

\begin{abstract}
Heterodyne polarimetry is based on the analysis of phases and polarization states of two frequency shifted cross-polarized waves, generated by Zeeman lasers and their analogs [1]. In semiconductor industry, manufacturing of memory chips depends on the width and aspect ratio of a great number of identical parallel vertical and horizontal word and bit address lines. Such a structure may be considered as a wire grid polarizer for visible optics, and it is reasonable to expect that polarimetry techniques may be efficient for detecting tiny variations in this type of structures on masks and wafers. Currently, both imaging and non-imaging modalities are considered as complementary inspection technologies. The talk will focus on instrumentation, theory, and experimental results of two different inspection tools: scanning polarimeters for mapping variations of critical dimensions over lithography masks and semiconductor wafers, and polarization-controlled dual-channel heterodyne microscope with super-resolution capabilities.
\end{abstract}

\section{Reference}

1. V Protopopov, Spinger Series in Optical Sciences, 149 (2009)

This is an Open Access article distributed under the terms of the Creative Commons Attribution-Noncommercial License 3.0, which permits unrestricted use, distribution, and reproduction in any noncommercial medium, provided the original work is properly cited. 